\title{
Bone mineral density, bone turnover markers, lean mass, and fat mass in Egyptian children with congenital adrenal hyperplasia
}

\author{
Soha M. Abd El Dayem¹, Ghada M. Anwar ${ }^{1}$, Hassan Salamaํ․ Ashraf F. Kamel ${ }^{1}$, Nahed Emara²
}

${ }^{1}$ Paediatric Department, National Research Centre, Cairo, Egypt

${ }^{2}$ Clinical Pathology Department, Cairo University, Cairo, Egypt

Submitted: 5 February 2009

Accepted: 16 May 2009

Arch Med Sci 2010; 6, 1: 104-110

DOI 10.5114/aoms.2010.13516

Copyright ๑ 2010 Termedia \& Banach

\begin{abstract}
Introduction: Aim of this paper is to assess bone mineral density (BMD) and body composition, by dual energy X-ray absorptiometry (DXA), and various markers of bone growth, in a group of children with congenital adrenal hyperplasia (CAH) on long-term glucocorticoid therapy.

Material and methods: A case-control study included thirty patients with $\mathrm{CAH}$ with different states of metabolic control. Their mean age was $7.5 \pm 4.2$ years. All patients are subjected to BMD using DXA at the neck of the femur and lumbar spine. A blood sample was taken for assessment of osteocalcin, osteoprotegerin, and procollagen type 1 , as markers of bone formation, as well as RANKL and urinary deoxypyridinoline (DPD), as markers of bone resorption. Results: We found no difference in BMD in patients and control subjects; however, patients showed significantly lower serum osteocalcin $(p=0.008)$ and osteoprotegerin $(p=0.0001)$ and significantly higher serum RANKL levels $(p=0.0001)$. Our results show that patients had significantly lower lean body mass $(p=0.005)$ and fat/lean ratio $(p=0.008)$ compared to matched controls. The duration of treatment showed a significant negative correlation with procollagen type $1(r=-0.49, p=0.02)$ and lean mass $\%(r=-0.43, p=0.04)$; however, it showed a significant positive correlation with total fat mass \% $(r=0.6, p=0.0006)$, and fat/lean ratio $(r=0.43, p=0.04)$. Dose of steroid had a significant positive correlation with BMI SDS $(r=0.4, p=0.02)$.

Conclusions: Bone mineral density is normal but bone turnover is low in patients with $\mathrm{CAH}$. There is an increase in fat/lean mass in patients with $\mathrm{CAH}$.
\end{abstract}

Key words: dual energy X-ray absorptiometry, bone mineral density, osteocalcin, osteoprotegerin, procollagen type 1 .

\section{Introduction}

Children with congenital adrenal hyperplasia (CAH) receive glucocorticoid therapy from the time of diagnosis, which may be at birth or even during fetal life. There is evidence that even modern glucocorticoid replacement therapy impacts on skeletal growth and maturation. Thus patients typically show some degree of short stature and delayed bone maturation during childhood [1].

In patients with $\mathrm{CAH}$ attributable to 21-hydroxylase deficiency, the synthesis of cortisol ( \pm aldosterone) is impaired. Consequently, the secretion of ACTH is increased, resulting in excess androgen production. Treatment of CAH consists of substitution of cortisol and aldosterone,

\section{Corresponding author:}

Nahed Emara, M.D, Ass. Prof. Clinical Pathology

Department

Faculty of Medicine

Cairo University, Egypt

E-mail:

dr.nahedemara@yahoo.com 
thereby preventing an adrenal crisis and suppressing adrenal androgen overproduction [2]. Glucocorticoids must be dosed carefully to avoid over-suppression (leading to growth retardation) and under-suppression (leading to androgen excess and reduction of final height) [3]. Glucocorticoid administration, even in substitution doses, may cause decreased bone mineral density (BMD) [4] and obesity [5]. Previous reports on BMD in $\mathrm{CAH}$ patients showed increased [6, 7], decreased [8-11], or normal [12-14] BMD. These reports differed with respect to age selections and glucocorticoid regimens. Body mass index (BMI) is found to be elevated in most $[8,9,11,13,15,16]$, but not all $[10,14]$ reports on CAH patients, but it was not clear whether this resulted from increased fat mass (as a result of glucocorticoid treatment, despite the low dose regimen) or increased lean mass (as a possible result of androgen excess) $[9,10]$.

Bone mass measurement defines mineral content per area of bone. In the laboratory, bone density by DXA is a very strong predictor of bone strength [17]. Biochemical markers of bone turnover have been developed to look at bone metabolism. They provide potentially useful information but cannot be used alone to diagnose osteoporosis, to determine the severity of the disease, or to select a specific therapy. Urinary deoxypyridinoline (DPD) and serum RANKL are markers of bone resorption, while osteocalcin, osteoprotegerin, and serum procollagen I carboxyterminal propeptide are markers of bone formation [18].

The aim of the current study was to assess BMD, lean mass, and fat mass, by DXA, and various markers of bone growth in a group of children with $\mathrm{CAH}$ who have received long-term glucocorticoid therapy under careful monitoring at our clinic.

\section{Material and methods}

It is a case-control study. Thirty patients with $\mathrm{CAH}$ were recruited from the Paediatric Department (Diabetes, Endocrine, and Metabolism Unit) at Cairo University Paediatric Hospital. We also studied 11 age- and sex-matched healthy controls who were included in our study. All subjects were healthy and appropriately physically active for their age; none was involved in competitive sport activities. Candidates were excluded if they had a history of chronic illness; they had one or more fractures; or they had taken any medication, hormone, vitamin preparation, or calcium supplements regularly.

Inclusion criteria were patients with $\mathrm{CAH}$ on treatment of more than 2 years' duration and exclusion criteria were newly diagnosed patients or treatment duration less than 2 years. The study included 27 patients with classic 21-hydroxylase deficiency, one patient with simple virilizing 21-hydroxylase deficiency and 2 patients with $3 \beta$ hydroxysteroid dehydrogenase deficiency. Twentyfour patients were diagnosed in the first year of life. The remaining 6 were diagnosed at the age of 2-9 years; they were simple virilizing $21 \mathrm{OH}$ deficiencies, late onset $3 \beta$ hydroxysteroid dehydrogenase deficiency, and late diagnosis of the classic forms. All had been treated from the onset of diagnosis with glucocorticoids and mineralocorticoids in salt losing forms or glucocorticoid in simple virilizing forms.

All patients were followed up regularly with biochemical, hormonal, and anthropometric measurements. The following data were obtained for patients and controls: age at diagnosis and start of therapy, duration of treatment, dose of glucocorticoid and mineralocorticoid for patients only, height and weight measured with a stadiometer and a clinical scale at the time of examination, body mass index (BMI) calculated as kilograms per metre squared, height SDS and weight SDS calculated with a growth calculator as previously described [19], BMI SDS expressed with data published by Cole et al. [20], pubertal assessment, LHRHanalogue treatment for patients only, and bone age assessed by X-ray of the left hand using the Greulich and Pyle method [21].

In the 21-hydroxylase deficient subjects, tight control was defined as a mean serum $170 \mathrm{H}$ progesterone value of all clinic visits of less than $10 \mathrm{nmol} / \mathrm{l}$, fair control was defined as a mean serum $170 \mathrm{H}$-progesterone value of $10-40 \mathrm{nmol} / \mathrm{l}$, together with normal levels of plasma rennin, and poor control was defined as a mean serum $170 \mathrm{H}$-progesterone value over $40 \mathrm{nmol} / \mathrm{l}$ [22]; these were noncompliant patients who also showed elevated levels of serum testosterone and plasma rennin [1]. The 2 patients with $3 \beta$-hydroxysteroid dehydrogenase deficiency were tightly controlled since their DHEA was below $5 \mathrm{nmol} / \mathrm{l}$ and normal plasma renin activity. All enrolled patients and controls were subjected (after their verbal consent) to:

1) bone mineral content (BMC, g), areal BMD (BMD, $\left.\mathrm{g} / \mathrm{cm}^{2}\right)$, fat mass, and lean mass were assessed by DXA (Norland - XR- 46, USA) at the lumbar spine (L1-L4) and the right femoral neck; absolute values were converted to Z-scores (standard deviations from the mean of a healthy age- and sex-matched reference population);

2) blood samples were collected by clean venipuncture into Vacutainer plane tubes under strict sterile conditions; $5 \mathrm{ml}$ of venous blood was obtained and put into a water bath for $15 \mathrm{~min}$ and centrifuged at $4000 \mathrm{rpm}$; serum was separated and kept at $-20^{\circ} \mathrm{C}$ for estimation of the different hormonal profiles as well as the following bone markers:

a) osteocalcin, using - ELISA kit (Biosource, Europe, S.A.) which is a quantitative sandwich enzyme-linked immunosorbent assay, 
b) serum osteoprotegerin using ELISA technique (Biovendor Laboratory Medicine, Inc., Czech Republic), Cat. No. RD 194003200; it is a biotin labelled antibody based sandwich enzyme immunoassay,

c) procollagen type 1 using Metra CICP assay; it is a sandwich enzyme immunoassay in a microtitre plate format utilizing a monoclonal anti-CICP antibody coated on the plate, a rabbit anti-CICP antiserum, a goat anti-rabbit alkaline phosphatase conjugate, and substrate to quantify the CICP in human serum,

d) serum RANKL levels by using ELISA kit, Biomedica group, Biomedica Medicine product, $\mathrm{GmbH}$ and $\mathrm{Co}$; it is an enzyme immunoassay for the quantitative determination of SRANKL in serum Cat. No. BI $20422 \mathrm{H}$,

e) serum testosterone was measured by chemiluminescence enzyme immunoassay (Immulite 2000 Diagnostic Products Los Angeles, CA, USA),

f) serum $17 \mathrm{OH}$ was measured by ELISA kit DRG (Germany) cat. no. EIA 1292 and it is based on the competition principle and micro-plate separation,

g) Delta 4 androstenedione, dehydroepiandrostenedione DHEA, and plasma renin were measured by Radioimmunoassay cat. no. IT 1322 Cis. Bioassay 30200 Bagnols/CIZA France; the procedure follows the basic principle of RIA, where there is competition between radioactive and non-radioactive antigen for a fixed number of antibody binding sites; the amount of 1.25 labelled antigen bound to the antibody is inversely proportional to their concentration; separation of the free and the bound antigen is achieved by decanting or aspirating the coated tubes;

3) urinary deoxypyridinoline (DPD) using Metra EIA DPD assay Cat. no. 8007; it was carried out by using preservative first morning void urine or secondary morning void urine; metra DPD assay is a competitive enzyme immunoassay in microtitre strip-well format utilizing a monoclonal anti-DPD antibody coated on the strip to capture DPD; DPD in the sample competes with conjugated DPD alkaline phosphatase for antibody and the reaction is detected with substrate; metra DPD results are corrected for urinary concentration by creatinine in urine.

\section{Statistical analysis}

Statistical Package for the Social Sciences (SPSS) program version 9.0 was used for analysis of data. Data were summarized as mean and SD. Nonparametric tests were used: Mann-Whitney $U$ test was used for analysis of 2 quantitative data and Kruskal-Wallis $U$ test for comparison of more than 2 quantitative variables. Friedman ANOVA was used to compare between the three groups of 21-hydroxylase deficiency patients (tight, fair, and poor control) and different parameters. Spearman correlation was done when appropriate.

\section{Results}

\section{Descriptive data of the study group}

Thirty patients with $\mathrm{CAH}$ were included in the study, mean age $7.5 \pm 4.2$ (range 3-18 years). Twentytwo were females and 8 were males. Among patients with 21-OH deficiency, 16 patients were on tight control (mean 17-OHP $2.4 \pm 2.3 \mathrm{nmol} / \mathrm{l}$ ), 9 patients showed fair control (mean 17-OHP $15.4 \pm 2.4 \mathrm{nmol} / \mathrm{l}$, normal PRA), and 3 patients showed bad control due to non-compliance (mean 17-OHP $47 \pm 4.3 \mathrm{nmol} / \mathrm{l}$, high PRA). Two patients with $3 \beta$ hydroxysteroid dehydrogenase deficiency were on tight control. Three of our patients received $\mathrm{LHRH}$ analogue due to central precocious puberty (1 with fair control and 2 with bad control). Descriptive data of the study group including their biochemical bone markers, body composition, and DXA results are shown in Table I.

\section{Comparison between patients and control} subjects

Patients included in the study showed significant lower lean body mass and higher total fat mass, abdomen fat $\%$, trunk fat $\%$, soft tissue fat mass $\%$ and fat/lean ratio compared to matched controls (Table II). Also, our patients showed significantly lower serum osteocalcin and osteoprotegerin, and significantly higher serum RANKL levels.

Effect of puberty, gender, control state, and treatment with $\mathrm{LHRH}$ analogue

Our data showed no significant differences between pre-pubertal $(n=9)$ and pubertal $(n=21)$ patients regarding their biochemical bone markers and DXA parameters except for BMC, which is higher in the pubertal group than in the prepubertal group $(1259.1 \pm 399.5 \mathrm{~g}$ and $840.3 \pm 458 \mathrm{~g}$, respectively, $p=0.05)$. Moreover, no significant difference was found between patients who received $\mathrm{LHRH}$ analogue and the rest of the patients in any of the DXA parameters or bone turnover markers. Our results show no significant differences between the three groups with different control state as well as between male and female patients regarding their biochemical markers and DXA results. 
Table I. Descriptive data of the study population

\begin{tabular}{|c|c|c|c|}
\hline & Mean \pm SD & Minimum & Maximum \\
\hline Age at start of therapy [years] & $1.86 \pm 4.13$ & 0.1 & 9 \\
\hline Duration of treatment [years] & $6.47 \pm 3.13$ & 2.4 & 14 \\
\hline Average dose of glucocorticoids [mg/M²/day] & $15.5 \pm 3.68$ & 5 & 21.5 \\
\hline Dose of mineralocorticoid [ $\mu \mathrm{g} / \mathrm{day}]$ & $0.11 \pm 4.1-02$ & 0.05 & 0.2 \\
\hline Height SDS & $-0.58 \pm 1.58$ & -3.7 & 2.3 \\
\hline Weight SDS & $7.14-03 \pm 1.79$ & -3 & 3.2 \\
\hline BMI SDS & $0.59 \pm 1.6$ & -3.2 & 3 \\
\hline Procollagen type 1 [ng/ml] & $325 \pm 150.8$ & 79 & 716 \\
\hline Osteocalcin [ng/ml] & $21.1 \pm 28.1$ & 0.1 & 75 \\
\hline Osteoprotegerin [pg/l] & $1.99 \pm 0.99$ & 0.3 & 3.6 \\
\hline Deoxypyridinoline [nmol/mmol creatinine] & $62.45 \pm 67$ & 1.13 & 285 \\
\hline RANKL [pmol/l] & $116.98 \pm 72.43$ & 40 & 290 \\
\hline BMD total $\left[\mathrm{gm} / \mathrm{cm}^{3}\right]$ & $0.64 \pm 9.9-2$ & 0.44 & 0.83 \\
\hline BMC total [gm] & $1144.86 \pm 447.6$ & 468 & 1954 \\
\hline Femur Z-score & $-0.184 \pm 0.66$ & -1.28 & 0.9 \\
\hline Vertebral Z-score & $0.28 \pm 0.76$ & -0.89 & 1.86 \\
\hline Total fat [\%] & $32.77 \pm 10.1$ & 12 & 47 \\
\hline Lean mass [\%] & $65.93 \pm 10.44$ & 51.25 & 87.28 \\
\hline Lean/fat ratio & $0.55 \pm 0.24$ & 0.15 & 0.95 \\
\hline Fat/lean ratio & $2.33 \pm 1.46$ & 1.1 & 6.86 \\
\hline Trunk fat [\%] & $28.2 \pm 3.34$ & 22.8 & 38.12 \\
\hline Abdomen fat [\%] & $16.5 \pm 3.89$ & 8.19 & 25.77 \\
\hline Soft tissue [\%] & $34.1 \pm 10.44$ & 12.72 & 48.75 \\
\hline
\end{tabular}

Table II. Comparison between patients and control subjects

\begin{tabular}{|lccc|}
\hline & Patients $(n=30)$ & Controls $(n=11)$ & $P$-value \\
\hline BMD [gm/cm ${ }^{3}$ ] & $0.64 \pm 1$ & $0.6 \pm 0.02$ & 0.05 \\
\hline BMC [gm] & $1144.0 \pm 447.6$ & $585.8 \pm 149.9$ & 0.06 \\
\hline Lean mass [\%] & $65.9 \pm 10.4$ & $76.1 \pm 5.6$ & 0.005 \\
\hline Fat mass [\%] & $32.8 \pm 10.1$ & $22.5 \pm 5.6$ & 0.006 \\
\hline Abdomen fat [\%] & $16.5 \pm 3.9$ & $13 \pm 5.1$ & 0.03 \\
\hline Trunk fat [\%] & $28.2 \pm 3.3$ & $21.6 \pm 8.3$ & 0.003 \\
\hline Soft tissue mass [\%] & $34.1 \pm 10.4$ & $23.9 \pm 5.6$ & 0.005 \\
\hline Fat/lean ratio & $0.6 \pm 0.2$ & $0.3 \pm 1$ & 0.008 \\
\hline Lean/fat ratio & $2.3 \pm 1.5$ & $3.4 \pm 1.2$ & 0.008 \\
\hline Osteocalcin [ng/ml] & $21.1 \pm 28.1$ & $49.4 \pm 34.5$ & 0.008 \\
\hline Osteoprotegerin [pg/l] & $2 \pm 1$ & $30.9 \pm 33$ & 0.0001 \\
\hline Procollagen type 1 [ng/ml] & $325 \pm 150.8$ & $376 \pm 218.5$ & 0.4 \\
\hline Deoxypyridinoline [nmol/mmol creatinine] & $62.4 \pm 67$ & $60.8 \pm 33.6$ & 0.3 \\
\hline RANKL [pmol/] & $117 \pm 72.4$ & $19.5 \pm 3.7$ & 0.0001 \\
\hline
\end{tabular}




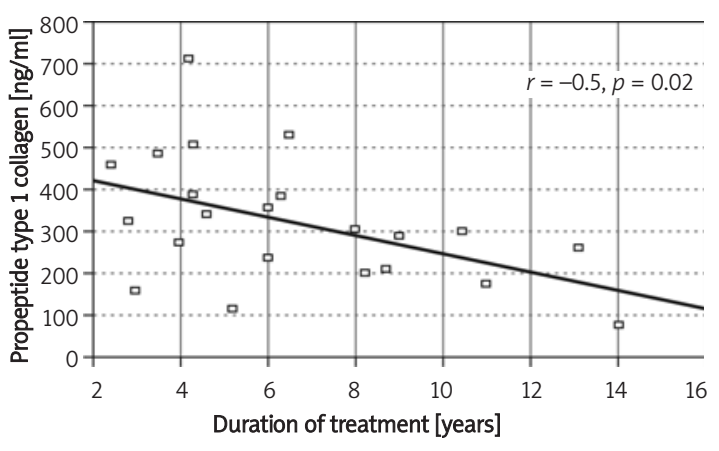

Figure 1. Relation of the duration of treatment in patients with $\mathrm{CAH}$ and procollagen type 1

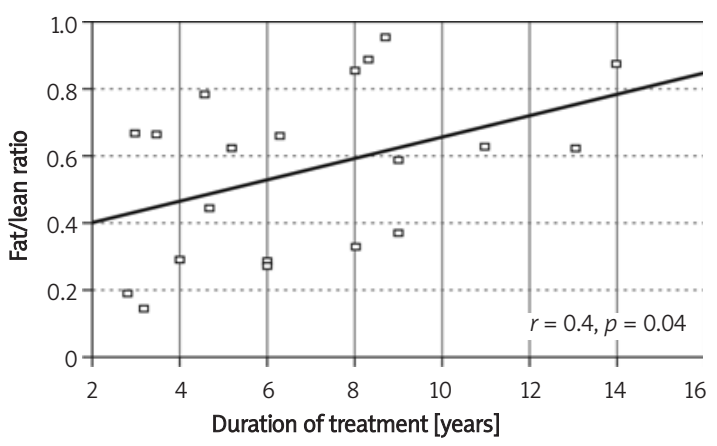

Figure 2. Relation of the duration of treatment in patients with $\mathrm{CAH}$ and fat/lean mass

\section{Effect of duration and dose of steroid treatment}

In our study, the duration of treatment showed a significant negative correlation with procollagen type $1(r=-0.49, p=0.02)$ (Figure 1$)$ and lean mass \% $(r=-0.43, p=0.04)$. However, it showed a significant positive correlation with abdomen fat $\%$ $(r=0.6, p=0.006)$, soft tissue $\%(r=0.43$, $p=0.04)$, total fat mass $\%(r=0.43, p=0.04)$, and fat/lean ratio $(r=0.43, p=0.04)$ (Figure 2). Dose of steroid showed a significant positive correlation with BMI SDS $(r=0.4, p=0.02)$.

\section{Correlation between BMI, biochemical bone markers, and DXA parameters}

BMI SDS showed a significant positive correlation with total fat $\%(r=0.7, p=0.001)$, fat/lean ratio $(r=0.72, p=0.0001)$, and soft tissue $\%$ $(r=0.7, p=0.0001)$. Accordingly, BMI SDS showed a negative correlation with lean mass \% $(r=-07, p=0.0001)$ and lean/fat ratio $(r=-0.6$, $p=0.004)$. Bone mineral density was not diminished in the distal femur (femur Z-score = $-0.184 \pm 0.66$ ) and lumbar spine (vertebral Z-score $=0.28 \pm 0.76$ ). No correlation was found between biochemical bone markers and any of the DXA parameters except for osteoprotegerin, a marker of bone formation, which showed a positive correlation with femur Z-score $(r=1, p=0.00001)$ and vertebral Z-score $(r=1, p=0.00001)$.

\section{Discussion}

In children with congenital adrenal hyperplasia, concerns have been raised that glucocorticoid replacement therapy might reduce growth and final height and might predispose to later osteoporosis $[2,16]$. In this study, we have shown that children and adolescents with $\mathrm{CAH}$ on long-term steroid therapy have normal bone mineral density; however, they have low serum osteocalcin, osteoprotegerin, and procollagen peptide of type 1 collagen and high serum RANKL levels, i.e. our patients have low bone formation markers and high bone resorption markers (Table II). We have found that vertebral Z-scores of our patients, which is a sensitive indicator to the effect of steroid therapy [23], are considered normal according to the World Health Organization [24]. Normal BMD in CAH patients has been reported earlier by other authors $[13,25,26]$, who found that adolescents with $\mathrm{CAH}$ had BMD values comparable with controls, despite glucocorticoid treatment. These authors hypothesized that negative effects of glucocorticoids on bone could be balanced by positive effects of androgens. Evidently, this may be true as long as glucocorticoid doses in $\mathrm{CAH}$ patients are in the low currently suggested dose [27]. Although our patients have low bone turnover, they have normal BMD. The normal bone status may be explained by the differential effects of glucocorticoids on growing bone, beneficial androgen effects, and other disease-specific factors [25]. The normal BMD that we observed in our patients does not rule out that these patients may develop glucocorticoid-induced osteoporosis at an older age. Lower lumbar and femoral neck BMD scores than controls were found in patients who had completed puberty [9]. As regards the bone turnover markers and their relation to $B M D$, our data are in agreement with Guo et al. [12], who found that BMD was not significantly decreased and that bone turnover was decreased in congenital adrenal hyperplasia patients. However, they measured serum osteocalcin and bone alkaline phosphatase to assess bone formation, whereas serum tartrateresistant acid phosphatase and urinary cross-linked $\mathrm{N}$-telopeptides of type I collagen were used to assess bone resorption. Also Sciannamblo et al. [28] found that bone resorption markers were higher in patients with $\mathrm{CAH}$.

In our clinic a protocol of regular monitoring of steroid dose, growth, serum $170 \mathrm{H}$-progesterone, testosterone, and plasma renin activity were followed. The average dose of hydrocortisone and mineralocorticoid was $15.5 \pm 3.68 \mathrm{mg} / \mathrm{M}^{2} /$ day and 0.11 $\pm 4.1-02 \mu \mathrm{g} /$ day, respectively. Although we had patients with different control states as previously mentioned, no significant difference between these groups regarding their BMD, BMC, body 
composition, and bone turnover markers was found. Moreover, the dose of steroid replacement did not show any significant relation to BMD or $B M C$ or any of the bone turnover markers. Girgis and Winter [1] found no significant difference between the three groups of metabolic control (tight, fair, and bad) regarding their vertebral BMD and serum osteocalcin, procollagen peptide, or collagen C-terminal telopeptide, or in urinary aminoterminal telopeptide. Mora et al. [13], Fleischman et al. [25], and Sciannamblo et al. [28] found that BMD measurements and bone turnover markers did not correlate with actual glucocorticoid dose or mean dose over the previous 7 years, which is consistent with our results. However, when glucocorticoids are given at higher doses, this will cause increased bone resorption and inhibition of bone formation, leading to reduced BMD. Previous reports showed that decreased $B M D$ in adult $\mathrm{CAH}$ patients was associated with glucocorticoid overdosing [9, 10]. Bachelot et al. [29] found that hydrocortisone dose was negatively correlated with the BMD T-score at the femoral neck.

As in earlier studies, we found that BMI was significantly higher in $\mathrm{CAH}$ patients compared with controls [10, 21, 29]. As regards body composition, our patients showed higher fat content than controls, which is related to the dose of steroid and duration of treatment. The increased fat mass could be the result of glucocorticoid use; other possible explanations for the increased fat mass could be adrenomedullary dysfunction with decreased catecholamine secretion, as recently described in $\mathrm{CAH}$ patients. Additionally, children with $\mathrm{CAH}$ had increased leptin levels and decreased insulin sensitivity, which has been ascribed to their adrenomedullary dysfunction.

In conclusion, children and adolescents with congenital adrenal hyperplasia have low bone turnover, normal BMD, high body fat content, and low lean mass. We recommend follow-up of the bone mineral density of these patients as they might be predisposed to later osteoporosis. In addition, we recommend further studies to clarify the role of calcium supplementation in $\mathrm{CAH}$ patients and study the diet provided to these patients to avoid overweight and obesity.

\section{References}

1. Girgis R, Winter JS. The effects of glucocorticoid replacement therapy on growth, bone mineral density, and bone turnover markers in children with congenital adrenal hyperplasia. J Clin Endocrinol Metab 1997; 82: 3926-9.

2. Stikkelbroeck NM, Oyen WG, van der Wilt GJ, Hermus RM, Otten BJ. Normal bone mineral density and lean body mass, but increased fat mass, in young adult patients with congenital adrenal hyperplasia. J Clin Endocrinol Metab 2003; 88: 1036-42.
3. Merke DP, Bornstein SR, Avila NA, Chrousos GP. NIH conference. Future directions in the study and management of congenital adrenal hyperplasia due to 21-hydroxylase deficiency. Ann Intern Med 2002; 36: 320-34.

4. Zelissen PM, Croughs RJ, van Rijk PP, Raymakers JA. Effect of glucocorticoid replacement therapy on bone mineral density in patients with Addison disease. Ann Intern Med 1994; 120: 207-10.

5. Kemink SA, Frijns JT, Hermus AR, Pieters GF, Smals AG, van Marken Lichtenbelt WD. Body composition determined by six different methods in women bilaterally adrenalectomized for treatment of Cushing's disease. J Clin Endocrinol Metab 1999; 84: 3991-9.

6. Speiser PW, New MI, Gertner JM. Increased bone mineral density in congenital adrenal hyperplasia. Pediatr Res 1993; 33: S81 (Abstract 465).

7. Arisaka O, Hoshi M, Kanazawa S, et al. Effect of adrenal androgen and estrogen on bone maturation and bone mineral density. Metab Clin Exp 2001; 50: 377-9.

8. Jääskeläinen J, Voutilainen R. Bone mineral density in relation to glucocorticoid substitution therapy in adult patients with 21-hydroxylase deficiency. Clin Endocrinol (Oxf) 1996; 45: 707-13.

9. Hagenfeldt K, Ritzen EM, Ringertz H, Helleday J, Carlström K. Bone mass and body composition of adult women with congenital virilizing 21-hydroxylase deficiency after glucocorticoid treatment since infancy. Eur J Endocrinol 2000; 143: 667-71.

10. Cameron FJ, Kaymakci B, Byrt EA, Ebeling PR, Warne GL, Wark JD. Bone mineral density and body composition in congenital adrenal hyperplasia. J Clin Endocrinol Metab 1995; 80: 2238-43.

11. Paganini C, Radetti G, Livieri C, Braga V, Migliavacca D, Adami S. Height, bone mineral density and bone markers in congenital adrenal hyperplasia. Horm Res 2000; 54: 164-8.

12. Guo CY, Weetman AP, Eastell R. Bone turnover and bone mineral density in patients with congenital adrenal hyperplasia. Clin Endocrinol (Oxf) 1996; 45: 535-41.

13. Mora S, Saggion F, Russo G, et al. Bone density in young patients with congenital adrenal hyperplasia. Bone 1996; 18: $337-40$.

14. Gussinye M, Carrascosa A, Potau N, et al. Bone mineral density in prepubertal and in adolescent and young adult patients with the salt-wasting form of congenital adrenal hyperplasia. Pediatrics 1997; 100: 671-4.

15. Cornean RE, Hindmarsh PC, Brook CG. Obesity in 21hydroxylase deficient patients. Arch Dis Child 1998; 78: 261-3.

16. Helleday J, Siwers B, Ritzen EM, Carlström K. Subnormal androgen and elevated progesterone levels in women treated for congenital virilizing 21-hydroxylase deficiency. J Clin Endocrinol Metab 1993; 76: 933-6.

17. Zebrowska A, Waszczykowska E. Osteopnia and osteoporosis in patients with dermatitis herpetifomis free diet. Arch Med Sci 2007; 3: 252-6.

18. Rosen JF The epidemiology and pathogenesis of osteoporosis. In: endotext.com. Chapter11. 2004. Available at: www.endotext.com.

19. Karlberg J, Albertsson-Wikland K. Growth in full-term small-for-gestational-age infants: from birth to final height. Pediatr Res 1995; 38: 733-9.

20. Cole TJ, Freeman JV, Preece MA. Body mass index reference curves for the UK. Arch Dis Child 1995; 73: 25-9.

21. Greulich WW, Pyle SI. Radiographic atlas of skeletal development of the hand and wrist. $2^{\text {nd }}$ ed. Palo Alto, CA: Stanford University Press 1959. 
22. Bachelot A, Chakhtoura Z, Rouxel A, Dulon J, Touraine P. Hormonal treatment of congenital adrenal hyperplasia due to 21-hydroxylase deficiency. Ann Endocrinol 2007; 68: 274-80.

23. Burckhardt P. Corticosteroids and bone: a review. Horm Res 1984; 20: 59-64.

24. Homik J, Hailey D. Bone density measurement: a health technology report, HTA. Available at: www.Alberta.com. 1999.

25. Fleischman A, Ringelheim J, Feldman HA, Gordon CM. Bone mineral status in children with congenital adrenal hyperplasia. J Pediatr Endocrinol Metab 2007; 20: 227-35.

26. Christiansen P, Mølgaard C, Müller J. Normal bone mineral content in young adults with congenital adrenal hyperplasia due to 21-hydroxylase deficiency. Horm Res 2004; 61: 133-6.

27. Gussinye M, Carrascosa A, Potau N, et al. Bone mineral density in prepubertal and in adolescent and young adult patients with the salt-wasting form of congenital adrenal hyperplasia. Pediatrics 1997; 100: 671-4.

28. Sciannamblo M, Russo G, Cuccato D, Chiumello G, Mora SJ. Reduced bone mineral density and increased bone metabolism rate in young adult patients with 21-hy droxylase deficiency. J Clin Endocrinol Metab 2006; 91: 4453-8.

29. Bachelot A, Plu-Bureau G, Thibaud E, et al. Long-term outcome of patients with congenital adrenal hyperplasia due to 21-hydroxylase deficiency. Horm Res 2007; 67: 268-76. 